\title{
Intra-osseous calcifying tendinitis of the infraspinatus tendon with erosion into the greater tuberosity
}

${ }^{1}$ Dr PH Henning MBChB(Pret)

${ }^{2}$ Dr R Truter MBChB, MMed(Rad)(D)(Stell)

${ }^{3}$ Dr M Boeyens MBChB, MMed(Orth)(D)(Pret)

${ }^{1,2}$ Prof S Andronikou MBBCh(Wits), FCRad(Diag)(SA), FRCR(Lond), PhD(UCT)

${ }^{1}$ Dr FE Suleman FCRad(D)(SA), MMed(Rad)(D)(Medunsa)

${ }^{1}$ Department of Radiology, University of Pretoria, Pretoria, South Africa

${ }^{2}$ Drs Schnettler, Corbett and Partners, Cape Town, South Africa

${ }^{3}$ Private practice, Cape Town, South Africa

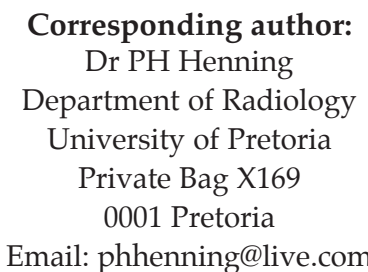

Tel: +27 (12) 354 2406; +27 (83) 4045000

\begin{abstract}
Calcific tendinitis is a condition characterised by the abnormal deposition of calcium hydroxyapatite crystals within the tendons of the rotator cuff. The characteristic appearance is that of acute or chronic shoulder pain combined with radiographic evidence of calcifications within the rotator cuff tendons. It is a specific disease entity which needs to be differentiated from other causes of rotator cuff calcium deposition.

We present a case of calcific tendinitis with secondary erosion at the humeral insertion of the infraspinatus tendon. Conventional radiography proved unsuccessful in the initial diagnosis of the condition. Advanced modalities including both computed tomography (CT) and magnetic resonance imaging (MRI) proved to be both diagnostic and pivotal in the further management of the patient.
\end{abstract}

Key words: rotator cuff, infraspinatus, calcific tendinitis, MRI, CT scan

\section{Introduction}

The common clinical presentation of a painful shoulder paired with the radiological findings of rotator cuff calcifications will invariably lead to the inclusion of calcific tendonitis in the differential diagnosis. It is important to recognise that calcific tendonitis refers to a specific pathological entity which requires a definitive diagnosis in order to facilitate the appropriate treatment.

Rotator cuff calcifications are a common entity, occurring in $7 \%$ of patients presenting with clinically significant shoulder pain. The median age of onset is in the fourth to fifth decade of life and there is a marginal increase in incidence among females.
There is a strong association with endocrine disorders, mainly disorders of thyroid and oestrogen metabolism. Other associated risk factors include excessive alcohol use and obesity. ${ }^{1,2}$ Concomitant injuries such as a rotator cuff tears are commonly associated with the condition and thus may conceal the diagnosis.

As in our case report, the calcifications in calcific tendonitis can be intra-osseous with erosions that may simulate an aggressive or benign skeletal neoplasm. ${ }^{3}$

Calcific tendonitis refers to a specific pathological entity which requires a definitive diagnosis in order to facilitate the appropriate treatment 


\section{Case report}

We present the case of a 55-year-old female referred with chronic shoulder pain. Conventional plain film imaging was the initial imaging modality performed. Standard shoulder views did not demonstrate any remarkable soft tissue calcifications to suggest the diagnosis. A subtle erosion at the tendon insertion on the greater tuberosity was retrospectively observed following further evaluation with CT and MRI (Figure 1).

Axial CT scan images revealed multiple specks of calcifications in the distal infraspinatus tendon. Erosion and sclerosis of the humeral head at the site of the tendon insertion were also demonstrated. Coronal reconstructed images demonstrated the subtle nature of the distal infraspinatus calcifications, which in all probability did not yield enough attenuation to be clearly visible on the AP radiographs (Figure 2a and $b$ ).

Axial MRI PD FS images demonstrated heterogeneous signal changes within the distal infraspinatus tendon in keeping with calcifications and oedema. The tendon was intact. Low signal changes were also visible in the humeral head, at the site of the infraspinatus tendon insertion, in keeping with erosion and sclerosis. Bone oedema surrounded the tendon insertion (high signal on FS images). Gradient echo (GE) images demonstrated blooming in the distal infraspinatus tendon which confirmed the presence of calcifications. The findings were in keeping with the diagnosis of calcific tendinopathy with intra-osseous extension (Figure 3).

\section{Discussion}

Calcific tendinitis is a recognised specific entity which warrants differentiation from other causes of intratendinous calcium deposition. There is still debate regarding the precise aetiology of the disease. The most accepted postulation regarding the pathogenesis of calcific tendinitis is that of hypoxia within a critical area of a tendon, followed by fibrocartilaginous metaplasia which will eventually calcify. The other hypotheses postulate that calcific depositions occur secondary to localised degeneration and avascularity within the distal segment of the affected tendon. Calcifications then develop secondary to necrotic tissue within the zone of avascularity. ${ }^{3}$

Although calcific tendinitis most frequently occurs in a single tendon, namely the supraspinatus tendon, involvement of multiple tendons of the rotator cuff may occur. The infraspinatus tendon, as with our case report, is the second most common site of reported calcific tendinitis, with the subscapularis tendon third. The most commonly affected locations include the critical zone of the supraspinatus tendon, the lower third of the infraspinatus tendon and the pre-insertional fibres of the subscapularis tendon. It is important to note that calcific deposits can also be found in numerous other tendons in the body. ${ }^{3}$
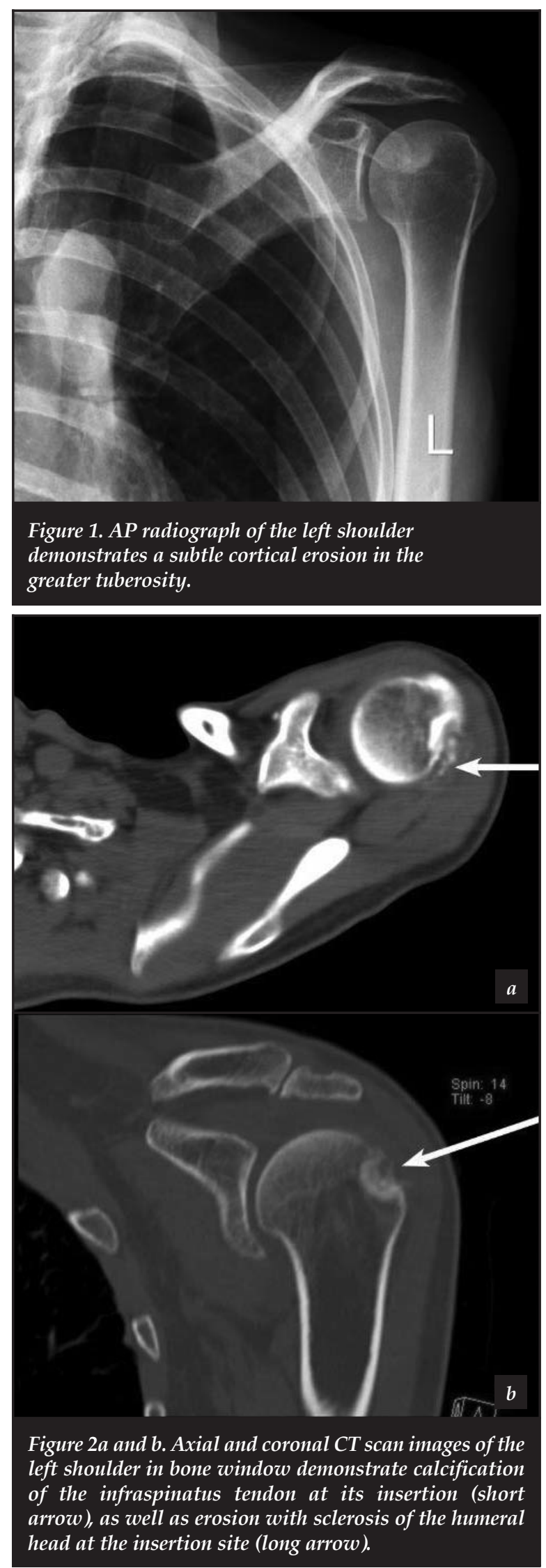


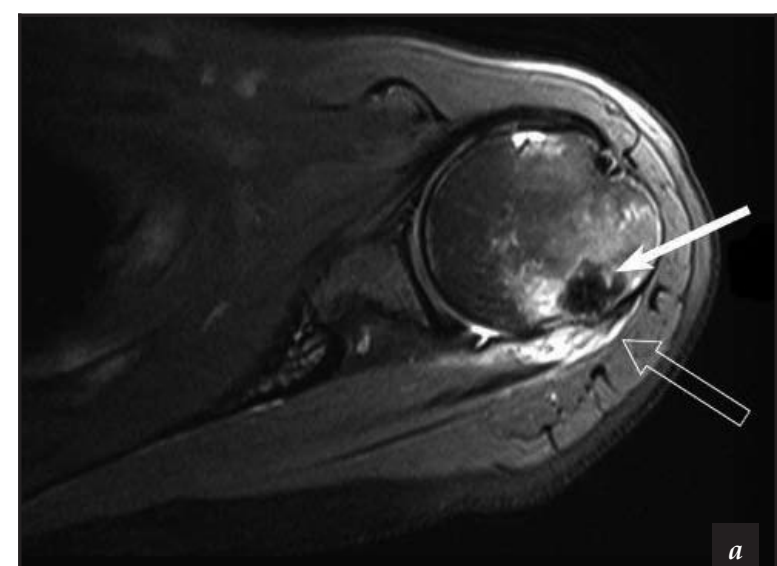

b

Figure $3 a$ and $b$. Axial MRI images of the left shoulder

3a: PD FS MRI demonstrates heterogeneous signal of the distal infraspinatus tendon (open arrow) in keeping with calcification and surrounding oedema within the tendon. There is also low signal within the humeral head at the site of tendon insertion (closed arrow) in keeping with erosion and sclerosis. The surrounding high signal indicates the presence of bone oedema.

3b: GE MRI demonstrates the low signal calcification in the tendon better due to gradient 'blooming'.

The clinical presentation of calcific tendinitis relates to the chronological deposition of calcium. First there is the formative phase in which fibrocartilaginous transformation occurs, followed by the resting phase in which there is no change in the size of calcifications.

The resting phase may be followed by the resorptive phase in which there is resorption of calcium with an inflammatory reaction. The typical clinical manifestation of subacute low-grade shoulder pain demonstrates a progressive increase throughout these stages.

The peak symptomatic presentation appears to be within the resorptive stage wherein acute inflammatory changes cause an increase in intra-tendinous pressure with sharp, localised acute pain that limits shoulder movement. $^{4}$
Conventional radiography remains the initial modality of choice for the evaluation of calcific tendinitis in order to assess for the presence of extra-cortical calcifications within the rotator cuff position. The French Arthroscopy Society Classification system is based on the morphological description of the calcifications involved; this includes solid calcifications (seen in $50 \%$ of patients), stippled calcifications (in 25\%) and amorphous calcifications (seen in 20\%). In 5\% of reported cases soft-tissue calcifications were not detected or characterised by conventional radiographs. Traditionally, elongated or comet-tail shaped intratendinous soft-tissue calcifications have been described but are only present in $50 \%$ of cases. In our case study no extra-cortical calcifications were apparent on the initial radiographs. Unfortunately the classification does not include intra-osseous osteolytic calcifications and it has been proposed that it should be included as a separate morphological entity in the classification of calcific tendonitis. ${ }^{5}$

Various studies recommend ultrasound as a sensitive modality for the detection of calcium deposits. Calcific plaque morphology, increased flow on power Doppler and widening of the subacromial-subdeltoid bursa were the most useful ultrasound findings. These findings correlated well with patient symptoms in the evaluation of calcific tendinitis. ${ }^{6}$ Ultrasound is also beneficial in pre- and postoperative evaluation. Ultrasound-guided marking of calcific deposits appears to significantly improve the clinical outcome of arthroscopic surgery. The use of ultrasound in our case study is limited by the inability to detect the intraosseous extent of the calcifications. The use of CT or MRI in cases of suspected intra-osseous deposits has been advocated because the failure of recognising the complete extent of the calcifications may lead to incomplete removal thereof. $^{5}$

Conventional radiography remains the initial modality of choice for the evaluation of calcific tendinitis

MRI findings include low signal globular masses within the distal ends of the rotator cuff tendons. The calcific depositions are of low signal on all sequences. The calcifications may be surrounded by high signal changes within the tendon, secondary to oedema and inflammation. These classical findings were present in our case study. Other MRI changes described in calcific tendinitis include T1 contrast enhancement secondary to peri-lesional inflammation. Sparing of the articular cartilage will be present on MR arthrography.

The main differential is degenerative calcification in a torn tendon. The chemical composition of these calcifications is different from those found in calcific tendinitis, and the size of the calcifications tends to be much larger than those found with degeneration. Other differentials include loose bodies with or without arthritis and osteochondromatosis. The hallmark of calcific tendinitis is the typical association with an intact rotator cuff. This finding radiologically differentiates calcific tendinitis from degenerative calcification from a torn tendon. ${ }^{3}$ 
Intra-osseous extension of the calcifications is an important entity to recognise since they will change the management of a patient. Cortical erosions are the most common manifestation of osseous involvement and have been found in all reported cases of intra-osseous deposition. CT and MRI can successfully detect cortical involvement not readily identified on conventional radiographs, as demonstrated in our case report. The osteolytic appearance of intra-osseous calcific depositions may mimic a tumour and MRI is pivotal in the differentiation of the causes. Porcellini et al. reported a $100 \%$ correlation between MRI and arthroscopically detected intra-osseous lesions. The only distinguishing characteristic of osteolytic calcifications was the size of the calcifications. The mean size of the calcification with intra-osseous extension was found to be larger than calcification without osseous extension. This finding was also demonstrated in the subsequent arthroscopic treatment of our patient. ${ }^{8}$

Calcific tendonitis is generally a self-limiting condition, and thus asymptomatic tendinitis does not require treatment. With mild symptoms conservative management with physical therapy and a short course of oral non-steroidal anti-inflammatory drugs appear to be effective. ${ }^{1,3}$ Advanced treatment options include needling under ultrasound guidance, high-energy extracorporeal shock-wave therapy and arthroscopy. Invasive treatment is indicated in patients affected by chronic and persistent calcific tendinitis who did not benefit from the nonsurgical treatment options after a period of at least 6 to 12 months. Arthroscopic treatment is also preferable when intra-osseous extension is demonstrated and has been found to improve both the degree of and time to functional recovery. ${ }^{8}$

\section{Conclusion}

Intra-osseous calcific tendinitis localised to the infraspinatus tendon is an uncommon presentation of a common condition. Calcific tendonitis should always be included in the differential of a patient presenting with shoulder pain and radiographic proof of tendon calcifications. Our case study highlights the fact that conventional radiography may prove unreliable in establishing the diagnosis, especially with calcifications localised to a single rotator cuff tendon, such as with the infraspinatus tendon, in our case study. Conventional radiography may also underestimate the extent and size of the disease process.
The early detection of osteolytic lesions secondary to calcific tendinitis is predictive of the degree and time to functional recovery. Advanced imaging studies, especially MRI and CT proved to be both reliable in establishing the diagnosis and have been found to be a good predictor of the expected findings during arthroscopic evaluation and treatment.

Conflict of interest statement: None of the authors have a conflict of interest relating to the subject matter.

\section{References}

1. Speed CA, Hazleman BL. Calcific tendinitis of the shoulder. N Engl J Med 1999;340(20):1582-84.

2. Harvie P, Pollard TCB, Carr AJ. Calcific tendinitis: Natural history and association with endocrine disorders. J Shoulder Elbow Surg 2007;16:169-73.

3. Flemming DJ, Murphey MD, Shekitka KM, Temple HT, Jelinek JJ, Kransdorf MJ. Osseous Involvement in calcific tendinitis: a retrospective review of 50 cases. AJR Am J Roentgenol 2003;181:965-72.

4. Uhthoff HK, Löhr JF. Calcifying tendinitis. In: Rockwood CA, Matsen FA, editors. The Shoulder. Volume 2. 2nd ed. Philadelphia: Saunders. 1998. pp989-1008

5. Seyahi A, Demirhan M. Arthroscopic removal of intraosseous and intratendinous deposits in calcifying tendinitis of the rotator cuff. Journal of Arthroscopic and related surgery 2009;25(6):590-96.

6. Le Goff B, Berthelot JM, Guillot P, Glemarec J, Maugars Y. Assesment of calcific tendonitis of rotator cuff by ultrasonography: comparison between symptomatic and asymptomatic shoulders. Joint Bone Spine 2010;77:258-63.

7. Kayser R. Hampf S. Seeber E. Heyde CE. Value of preoperative ultrasound marking of calcium deposits in patients who require surgical treatment of calcific tendinitis of the shoulder. Arthroscopy 2007;23:43-50

8. Porcellini G, Paladini P, Campi F, Pegreffi F. Osteolytic lesion in the greater tuberosity in calcific tendinitis of the shoulder. J Shoulder Elbow Surg 2009;18:210-15.

This article is also available online on the SAOA website (www.saoa.org.za) and the SciELO website (www.scielo.org.za). Follow the directions on the Contents page of this journal to access it. 